\title{
Probing the Thermochromic Phase
}

\section{Transition in $\mathrm{CuMoO}_{4}$ by EXAFS}

\section{Spectroscopy}

\author{
I. Jonane ${ }^{*}$, , A. Cintins ${ }^{1}$, A. Kalinko ${ }^{2}$, R. Chernikov ${ }^{3}$, A. Kuzmin ${ }^{1}$ \\ ${ }^{1}$ Institute of Solid State Physics, University of Latvia, Kengaraga street 8, LV-1063 Riga, Latvia \\ ${ }^{2}$ Department Chemie, Naturwissenschaftliche Fakultät, Universität Paderborn, Warburger Straße 100, D-33098 Paderborn, Germany \\ ${ }^{3}$ DESY Photon Science, Notkestraße 85, D-22607 Hamburg, Germany
}

Key words: $\mathrm{CuMoO}_{4}$, Mo K-edge, EXAFS, phase transition

${ }^{*}$ Corresponding author: e-mail inga.jonane@cfi.lu.lv, Fax: +371 67132778

Extended X-ray absorption fine structure (EXAFS) spectroscopy at the Mo K-edge was used to follow the thermochromic $\gamma$-to- $\alpha$ phase transition in copper molybdate $\left(\mathrm{CuMoO}_{4}\right)$. The temperature dependence (10-300 K) of the radial distribution function (RDF) for the Mo-O atom pairs in the first coordination shell of molybdenum atoms was reconstructed by the regularization-like method from the experimental EXAFS spectra. The analysis of the RDFs suggests that the transition occurs gradually within the two-phase coexistence range of 225-275 K. The local environment of molybdenum atoms transforms upon the $\gamma$-to- $\alpha$ phase transition from strongly distorted octahedral to less distorted tetrahedral coordination.

Copyright line will be provided by the publisher 


\section{Introduction}

Copper molybdate $\left(\mathrm{CuMoO}_{4}\right)$ has attracted considerable interest due to its thermochromic and piezochromic properties, which originate from the first order phase transition between the $\gamma$ and $\alpha$ phases. ${ }^{1-4}$ Materials with such properties have been widely studied as prospective components for temperature and/or shock sensors. ${ }^{4-7}$ The structural, optical, electrical and magnetic properties of $\mathrm{CuMoO}_{4}$ were studied previously. ${ }^{1-4,8,9}$ The temperature-pressure diagram for $\mathrm{CuMoO}_{4}$ was determined based on diffraction data. ${ }^{1}$

At ambient pressure and temperatures below $300 \mathrm{~K}$, copper molybdate exists in two phases (Figure 1): brownish-red $\gamma-\mathrm{CuMoO}_{4}$ and green $\alpha-\mathrm{CuMoO}_{4} \cdot{ }^{1}$ In addition to the colour change, the $\gamma$-to- $\alpha$ phase transition is accompanied by a volume increase of $12-13 \% .{ }^{1}$ Optical reflectivity measurements indicate that the transition occurs between $175 \mathrm{~K}$ and $260 \mathrm{~K} .{ }^{4}$ The low-temperature $\gamma$-CuMoO${ }_{4}$ phase has triclinic $P \overline{1}$ symmetry and is composed of distorted $\mathrm{CuO}_{6}$ and distorted $\mathrm{MoO}_{6}$ octahedra. ${ }^{8}$ The structure of $\alpha-\mathrm{CuMoO}_{4}$, which is being stable at room temperature, also has triclinic $P \overline{1}$ symmetry and is composed of distorted $\mathrm{CuO}_{6}$ octahedra, $\mathrm{CuO}_{5}$ square-pyramids and distorted $\mathrm{MoO}_{4}$ tetrahedra. ${ }^{10}$ The unit cell of $\mathrm{CuMoO}_{4}$ in both phases contains six formula units $(Z=6)$ with three non-equivalent copper and three non-equivalent molybdenum atoms, which have different local environments. ${ }^{1,8}$ Based on diffraction data, the $\gamma$-to- $\alpha$ phase transition is described as "pseudoreconstructive" with an extensive two phase coexistence range and hysteretic behaviour. ${ }^{1,6}$

The chromatic properties of $\mathrm{CuMoO}_{4}$ are explained based on the interplay between two optical absorption bands. The first band, located in the red spectral range below $\sim 1.8 \mathrm{eV}$, is related to the copper $3 \mathrm{~d}^{9} \rightarrow 4 \mathrm{p}^{3}$ or $\mathrm{d}$-d transition. ${ }^{2,4,11}$ The second band, located in the blue spectral range above $\sim 2.5 \mathrm{eV}$, is attributed to the oxygen-to-metal charge transfer band. ${ }^{2-4,11}$

Recently, we used X-ray absorption near edge structure (XANES) spectroscopy to follow the $\alpha$-to$\gamma$ and $\gamma$-to- $\alpha$ structural phase transitions in $\mathrm{CuMoO}_{4}$ upon cooling and heating, respectively. ${ }^{12}$ While only small variations in XANES were detected at the $\mathrm{Cu}$ K-edge, significant changes in the intensity of the pre-edge shoulder at the Mo K-edge were observed. These changes occur due to a transformation of the local environment of molybdenum atoms between distorted tetrahedral and distorted octahedral coordinations and can thus be used to follow the hysteretic behaviour of the phase transition. 


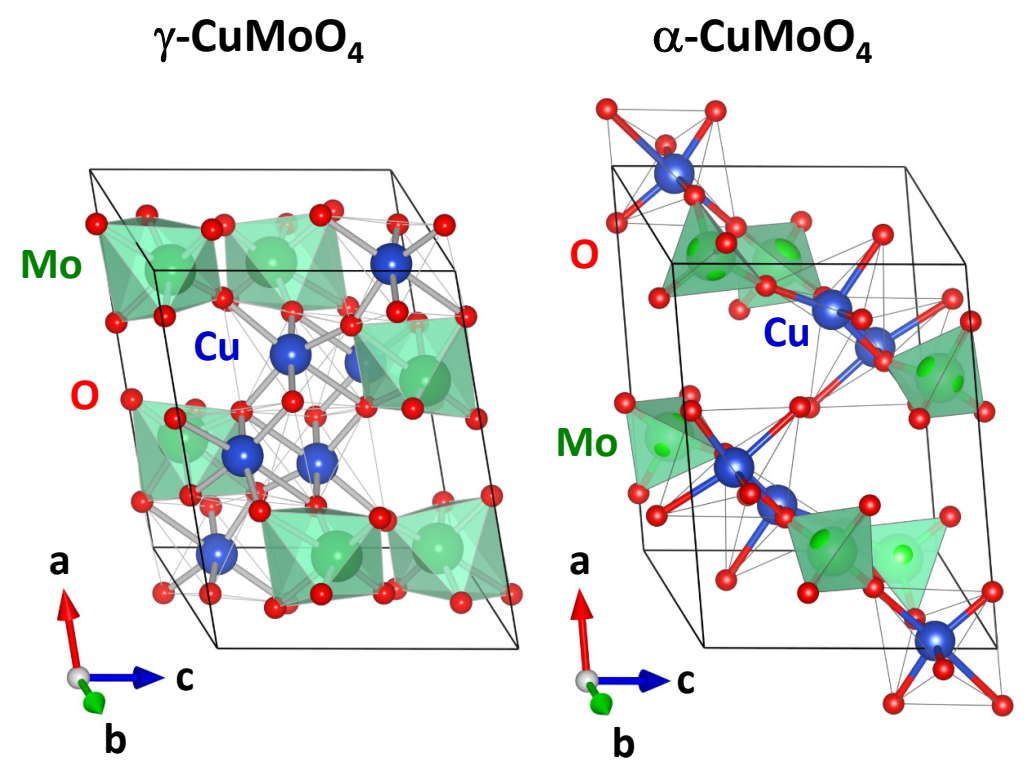

Figure 1 Crystal structures and unit cells of low-temperature $\gamma-\mathrm{CuMoO}_{4}$ and high-temperature $\alpha$-CuMoO 4 phases. ${ }^{1}$ $\mathrm{MoO}_{6}$ and $\mathrm{MoO}_{4}$ polyhedra are indicated in the $\gamma$ - and $\alpha$-phases, respectively. Small red balls are oxygen atoms, mediumsized blue balls are copper atoms, and large green balls are molybdenum atoms.

A deep understanding of the structure-thermochromic property relationship in $\mathrm{CuMoO}_{4}$ requires detailed information on the temperature dependence of the local atomic structure, which is encoded in the extended X-ray absorption fine structure (EXAFS). The reliable extraction of such infomation is not trivial in the case of materials with strong local distortions such as $\mathrm{CuMoO}_{4}$. Therefore, in this study, the advanced EXAFS analysis approach based on the regularisation method ${ }^{13}$ was used to extract the temperature dependence $(10-300 \mathrm{~K})$ of the radial distribution function (RDF) within the first coordination shell around molybdenum atoms. This approach is model independent and, thus, does not require any preliminary assumption on the coordination of molybdenum atoms. As a result, we were able to follow the variation of the molybdenum environment from octahedral to tetrahedral upon the thermochromic $\gamma$-to- $\alpha$ phase transition.

\section{Experimental Section}

Polycrystalline $\mathrm{CuMoO}_{4}$ was produced by heating a mixture of $\mathrm{CuO}$ and $\mathrm{MoO}_{3}$ powders at $650{ }^{\circ} \mathrm{C}$ in air for $8 \mathrm{~h}$. The as-synthesized sample had a green color corresponding to $\alpha-\mathrm{CuMoO}_{4}$, and its phase purity was confirmed by X-ray powder diffraction. 
Temperature-dependent X-ray absorption spectra were recorded at the Mo K-edge (20000 eV) in transmission mode at the HASYLAB/DESY PETRA-III P65 undulator beamline. The PETRAIII storage ring was operated at $E=6.08 \mathrm{GeV}$ and current $I=95 \mathrm{~mA}$ in top-up 40 bunch mode. Harmonic rejection was achieved by a Rh-coated silicon plane mirror, and monochromatic X-rays were produced by a fixed-exit Si (311) double-crystal monochromator. Two ionisation chambers were employed for the X-ray intensity measurements before and after the sample. An Oxford Instruments liquid helium flow cryostat was used to maintain the sample temperature. The sample was prepared from $\mathrm{CuMoO}_{4}$ powder, which was gently milled in an agate mortar and deposited on a Millipore membrane. The measurements were performed upon heating at twelve selected temperatures in the range from 10 to $300 \mathrm{~K}$. Each measurement was conducted after the sample temperature was stabilized for approximately 20 minutes.

\section{Data Analysis}

Temperature-dependent experimental Mo K-edge EXAFS $\chi(k) k^{2}$ spectra of $\mathrm{CuMoO}_{4}$ were extracted following the conventional procedure ${ }^{14}$ using the Athena package. ${ }^{15}$ The photoelectron wavenumber $k$ is defined as $k=\sqrt{2 m\left(E-E_{0}\right)} / \hbar$, where $m$ is the electron mass, $\hbar$ is Plank's constant, $E$ is the $\mathrm{X}$-ray photon energy and $E_{0}$ is the origin of the photoelectron kinetic energy.

The EXAFS spectra and their Fourier transforms (FTs) at selected temperatures are shown in Figure 2. The FTs were calculated in the $k$-space range of $2.5-14.5 \AA^{-1}$. Note that the FTs were not corrected for the backscattering phase shift of atoms; therefore, the positions of all peaks are displaced to smaller distances relative to their crystallographic values. Due to the low symmetry of $\mathrm{CuMoO}_{4}$, the contributions from coordination shells around molybdenum atoms overlap strongly and, therefore, no unique peak assignment can be done in the FTs beyond the first coordination shell. The broad shape of the first shell peak, located between 0.8 and $2.2 \AA$ in Figure 2, is due to the presence of three non-equivalent molybdenum atoms and a distortion of molybdenum-oxygen polyhedra.

The EXAFS signals due to the first coordination shell of molybdenum atoms (Figure 3) were isolated from the high-frequency outer shell contributions using the inverse FT in the range of $0.5-2.5 \AA$ and were used in the further analysis. Note that the EXAFS signals from oxygen atoms located in the 

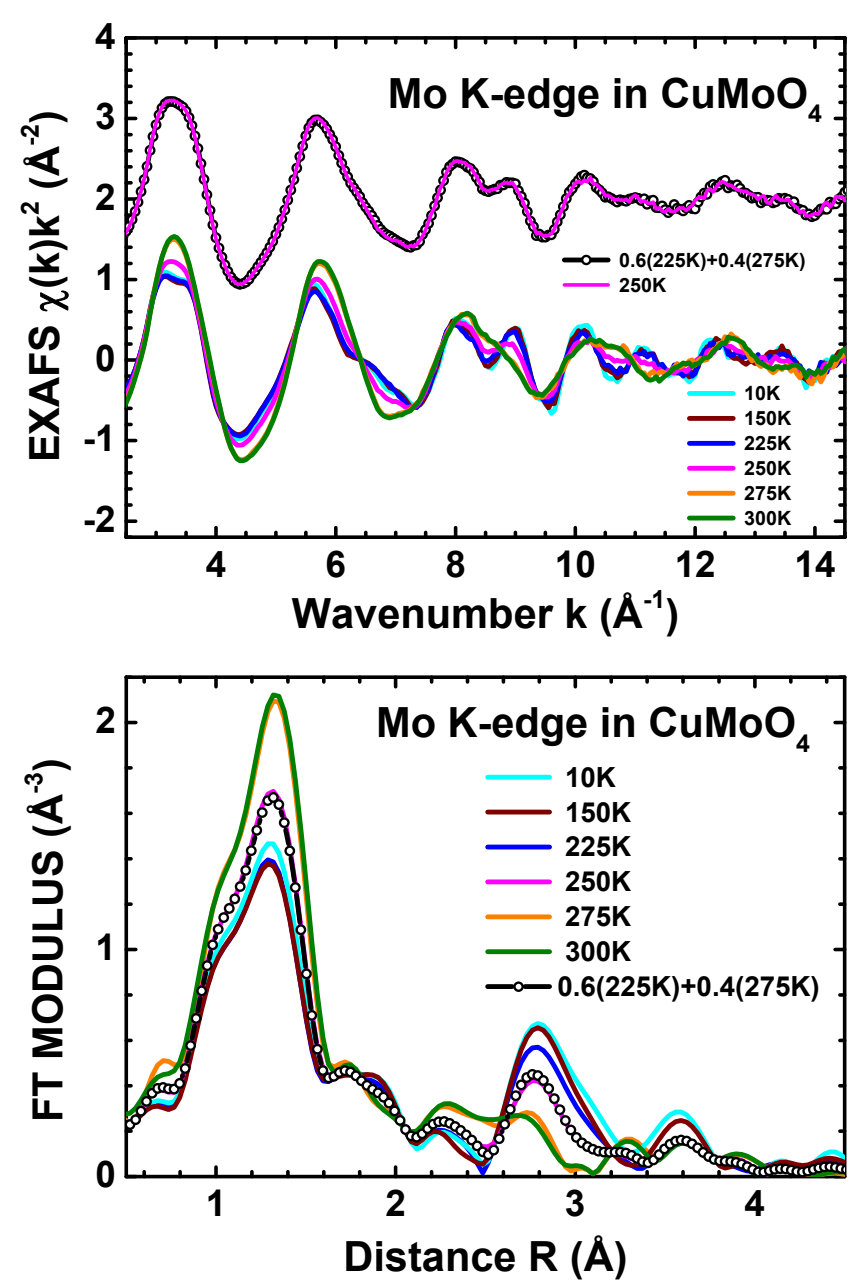

Figure 2 Temperature dependence of the selected experimental Mo K-edge EXAFS $\chi(k) k^{2}$ spectra (upper panel) and their Fourier transforms (lower panel, only the modulus is shown) for $\mathrm{CuMoO}_{4}$. A weighted sum of the two EXAFS spectra recorded at $225 \mathrm{~K}$ and $275 \mathrm{~K}$ is shown by open circles; it is compared with the experimental EXAFS spectrum measured at $250 \mathrm{~K}$ in the upper panel.

first shell are damped due to static distortions and thermal disorder. The same extended range in the $R$-space was used at all temperatures to reliably include contributions in the case of octahedral and tetrahedral environments of molybdenum atoms.

The change in the molybdenum environment from octahedral to tetrahedral coordination upon heating from 10 to $300 \mathrm{~K}$ strongly affects the Mo K-edge EXAFS spectra and their FTs (Figure 2). Modifications of the atomic structure occur in all coordination shells leading to a variation of the peaks in the FTs. Note that the analysis of distant coordination shells is challenging due to their strong overlap and the presence of multiple-scattering contributions. Such analysis requires the use of advanced techniques, such as, reverse Monte Carlo simulations, ${ }^{16,17}$ and is beyond the scope of the 


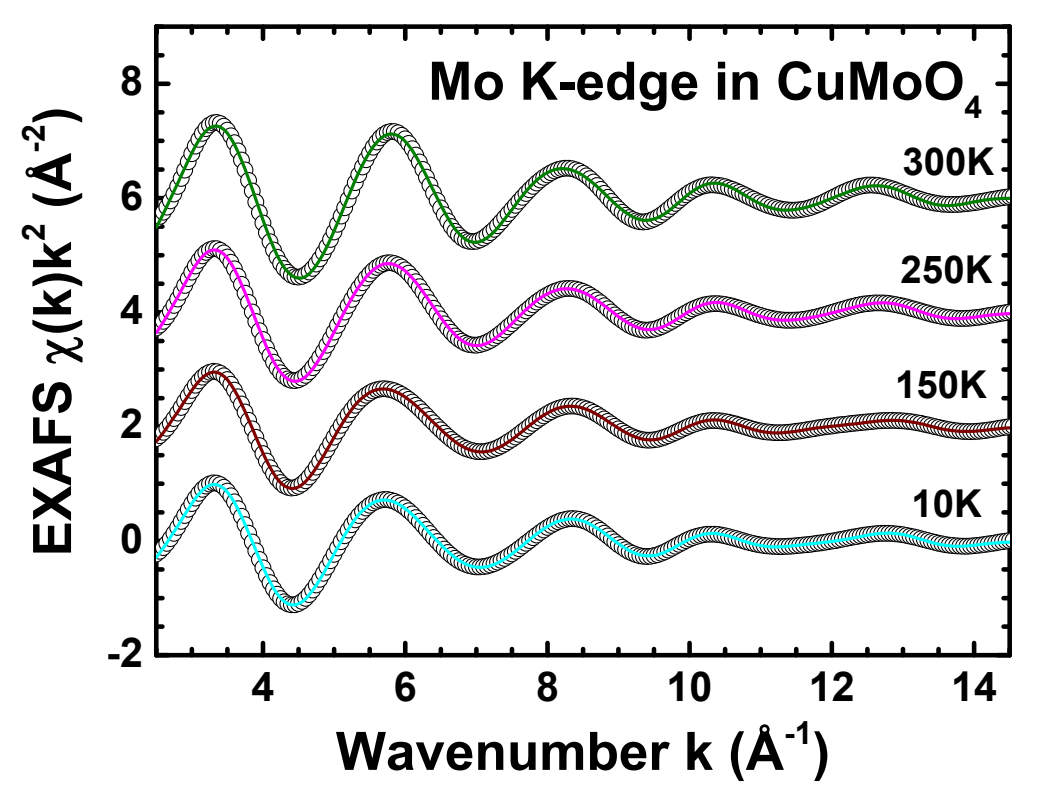

Figure 3 Comparison of the experimental (open circles) and calculated by the regularization-like method ${ }^{13}$ (solid lines) Mo K-edge EXAFS $\chi(k) k^{2}$ spectra for the first coordination shell of molybdenum atoms in $\mathrm{CuMoO}_{4}$ at four selected temperatures.

present study. Here, we limited our discussion to the first coordination shell of molybdenum atoms (the group of peaks in the FTs up to $\sim 2.2 \AA$ in Figure 2 ) because the change in their local environment is largely responsible for $\mathrm{CuMoO}_{4}$ optical properties.

Recently, we successfully used a linear combination method to describe the variation of the Mo K-edge XANES in $\mathrm{CuMoO}_{4}$ over a wide range of temperatures from 10 to $300 \mathrm{~K} .{ }^{12}$ We found that the XANES at each temperature can be described by the weighted sum of the two XANES spectra corresponding to pure $\gamma-\mathrm{CuMoO}_{4}$ and $\alpha-\mathrm{CuMoO}_{4}$ phases measured at $10 \mathrm{~K}$ and $300 \mathrm{~K}$, respectively. Such a simple approach works nicely for the XANES of $\mathrm{CuMoO}_{4}$ because it is weakly sensitive to thermal disorder. At the same time, this method cannot be applied to the treatment of EXAFS spectra due to the fact that their amplitude is strongly affected by the presence of thermal disorder, leading to strong oscillation damping at high- $k$ values. However, when EXAFS spectra at relatively close temperatures are available, the difference introduced by the thermal disorder is not as crucial. For example, it is possible to simulate the Mo K-edge EXAFS at $250 \mathrm{~K}$ as the weighted sum of the two EXAFS spectra recorded at $225 \mathrm{~K}(60 \%)$ and $275 \mathrm{~K}(40 \%)$ (Figure 2). 
To overcome the problem of thermal disorder, one can follow the temperature variation of structural parameters, whose determination is also complicated due to static distortions present in $\mathrm{CuMoO}_{4}$ in addition to thermal effects. Such a situation is best described in terms of distribution functions. Therefore, we employed a model independent approach based on the regularization-like method ${ }^{13}$ to obtain the temperature dependence of the radial distribution function (RDF) $G(R)(\mathrm{Mo}-\mathrm{O})$ in the first coordination shell of molybdenum atoms. This method has an advantage over conventional multicomponent fitting in the case of a strongly distorted local environment as one finds in tungstates and molybdates. ${ }^{18,19}$ Next, we took into account that the group of four nearest oxygen atoms, which are strongly bound to molybdenum atoms in both $\mathrm{CuMoO}_{4}$ phases, remains nearly at the same mean distance $(\sim 1.76 \AA$ ) but is sensitive to structural distortions reflected by the peak broadening in RDFs. Therefore, the analysis of this group can be used to follow the phase transition.

The EXAFS $\chi(k)$ from the first coordination shell is defined within the spherical-wave approxima$\operatorname{tion}^{20}$ as

$$
\chi(k)=S_{0}^{2} \int \frac{G(R)}{k R^{2}} f^{l}(\pi, k, R) \sin \left(2 k R+\phi^{l}(\pi, k, R)\right) d R
$$

where $S_{0}^{2}$ is the scale factor taking into account amplitude damping due to the multielectron effects, $f^{l}(\pi, k, R)$ is the backscattering amplitude of the photoelectron due to the atom located at a distance $R$ from the photoabsorber, and $\phi^{l}(\pi, k, R)=\varphi^{l}(\pi, k, R)+2 \delta_{l}(k)-l \pi$ is the phase shift containing contributions from the photoabsorber $2 \delta_{l}(k)$ and the backscatterer $\varphi^{l}(\pi, k, R)$ ( $l$ is the angular momentum of the photoelectron). The integration in Eq. (1) is performed over the first coordination shell range $\left[R_{\min }, R_{\max }\right]$. In this case, we used $R_{\min }=0.8 \AA$ and $R_{\max }=2.8 \AA$.

The theoretical backscattering amplitude $f^{l}(\pi, k, R)$ and phase shift $\phi^{l}(\pi, k, R)$ functions, used in the EXAFS simulations using Eq. (1), were calculated for the $\gamma-\mathrm{CuMoO}_{4}$ crystallographic structure ${ }^{1}$ using the self-consistent, real space multiple-scattering FEFF8.5L code. ${ }^{21,22}$ The complex energydependent exchange-correlation Hedin-Lundqvist potential ${ }^{23}$ was employed to account for inelastic effects. The obtained best-fits for selected temperatures are shown in Figure 3, and the corresponding RDFs $G(R)(\mathrm{Mo}-\mathrm{O})$ in the range of interest from $1.55 \AA$ to $2.6 \AA$ are presented in Figure 4 . The RDFs at $10 \mathrm{~K}$ and $300 \mathrm{~K}$ in all fitting range, supplemented with the crystallographic Mo-O distances, ${ }^{1}$ 


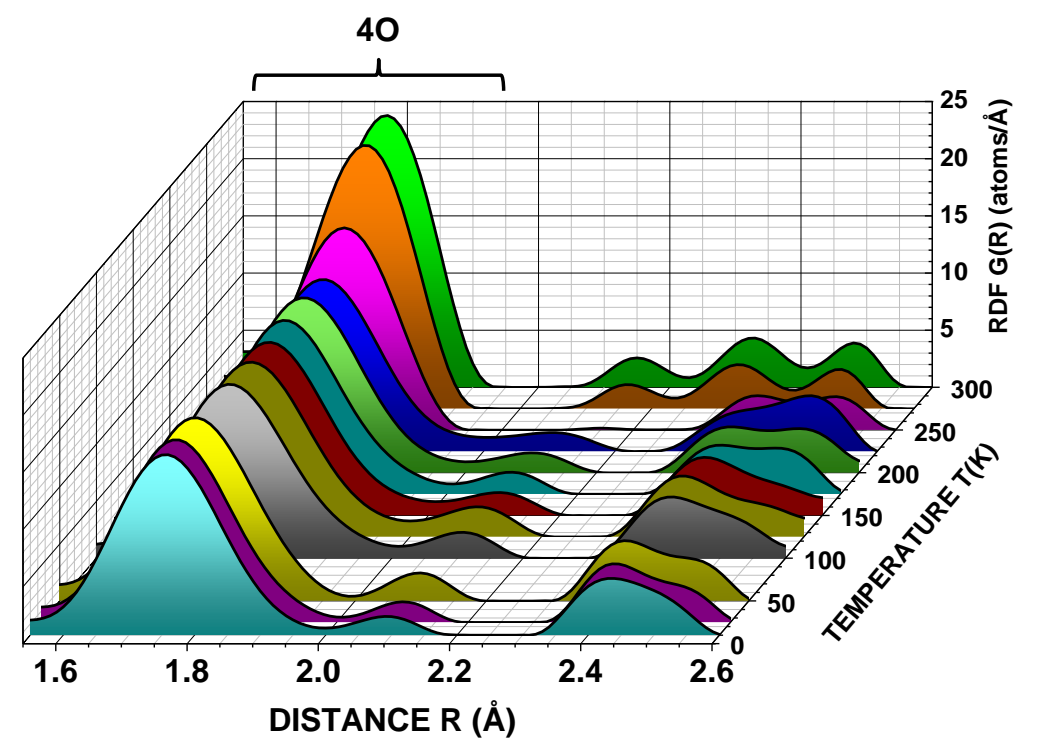

Figure 4 Temperature dependence of the radial distribution functions (RDFs) $G(R)(\mathrm{Mo}-\mathrm{O})$ in $\mathrm{CuMoO}_{4}$, obtained by the regularization-like method. ${ }^{13}$ The first peak due to the four nearest oxygen atoms is indicated.

are shown in Figure S1 in the Supporting Information. The first peak located in the range of 1.55$2.0 \AA$ corresponds to the group of four nearest oxygen atoms: their contribution dominates in both $\mathrm{CuMoO}_{4}$ phases and is therefore useful for following the phase transition. The two distant oxygen atoms of $\mathrm{MoO}_{6}$ octahedra in $\gamma-\mathrm{CuMoO}_{4}(T<250 \mathrm{~K})$ are also detectable at 2.0-2.6 $\AA$. Note that the RDFs $G(R)$ in $\alpha-\mathrm{CuMoO}_{4}(T>250 \mathrm{~K})$ contain several spurious peaks at $R>2.1 \AA$, which are due to some unfiltered multiple-scattering contributions generated within $\mathrm{MoO}_{4}$ tetrahedra. ${ }^{24}$

\section{Results and Discussion}

Twelve experimental Mo K-edge EXAFS spectra were measured upon heating $\mathrm{CuMoO}_{4}$ from 10 to $300 \mathrm{~K}$. The selected EXAFS spectra $\chi(k) k^{2}$ and their Fourier transforms are shown in Figure 2. The spectra change significantly upon the phase transition due to the structural transformations occurring in the first and distant coordination shells. One can distinguish three groups of EXAFS spectra: (i) the spectra below $225 \mathrm{~K}$ correspond to the pure $\gamma-\mathrm{CuMoO}_{4}$ phase, (ii) the spectra above $275 \mathrm{~K}$ correspond to the pure $\alpha-\mathrm{CuMoO}_{4}$ phase, and (iii) the spectrum at $250 \mathrm{~K}$ corresponds to a situation with two coexisting phases. Note that the EXAFS spectrum at $250 \mathrm{~K}$ can be well described by the weighted sum of the two EXAFS spectra corresponding to the $\gamma$-phase at $225 \mathrm{~K}$ and the $\alpha$-phase at $275 \mathrm{~K}$ with 


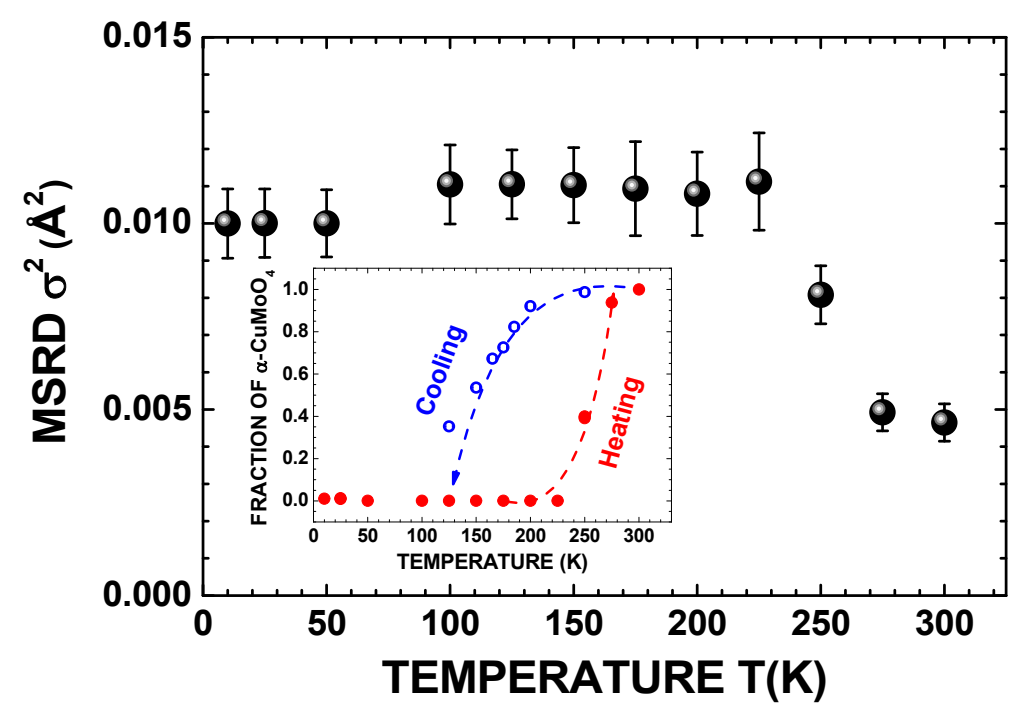

Figure 5 Temperature dependence of the mean-square relative displacement (MSRD) $\sigma^{2}$ for the four shortest Mo-O interatomic distances in $\mathrm{CuMoO}_{4}$. Inset: the phase transition hysteresis obtained from the Mo K-edge $\mathrm{XANES}_{\mathrm{N}} \mathrm{CuMoO} 4$ upon heating and cooling. ${ }^{12}$

weights of $60 \%$ and $40 \%$, respectively (Figure 2). This result agrees well with the linear combination analysis of the Mo K-edge XANES spectra (see the inset in Figure 5) in our previous study. ${ }^{12}$

The analysis of EXAFS spectra using the regularization-like method ${ }^{13}$ resulted in good agreement with the experimental data (Figure 3) and allowed us to obtain temperature-dependent RDFs $G(R)(\mathrm{Mo}-\mathrm{O})$ for the first coordination shell of molybdenum atoms (Figure 4).

According to the diffraction data, ${ }^{1} 12$ different Mo-O bonds $(1.737 \AA, 1.762 \AA, 1.783 \AA$, and $1.825 \AA$ for $\operatorname{Mo}(1) ; 1.706 \AA, 1.778 \AA, 1.782 \AA$, and $1.795 \AA$ for $\operatorname{Mo}(2) ; 1.701 \AA, 1.704 \AA, 1.837 \AA$, and $1.863 \AA$ for $\mathrm{Mo}(3)$ ) exist around three non-equivalent molybdenum atoms with tetrahedral coordination in $\alpha-\mathrm{CuMoO}_{4}$. Thus, the mean distance to the four nearest oxygen atoms is equal to $\sim 1.77 \AA$ with a standard deviation of $0.05 \AA$. In $\gamma-\mathrm{CuMoO}_{4}$ the coordination of molybdenum atoms is octahedral, and oxygen atoms are split into two groups of the four nearest and two distant atoms. ${ }^{1}$ Again, three non-equivalent molybdenum atoms exist, leading to 18 different Mo-O bonds: $1.690 \AA$, $1.776 \AA$, $1.875 \AA, 1.930 \AA, 2.132 \AA$, and $2.478 \AA$ for $\operatorname{Mo}(1) ; 1.683 \AA, 1.781 \AA, 1.881 \AA, 1.926 \AA, 2.105 \AA$, and $2.471 \AA$ for $\operatorname{Mo}(2) ; 1.701 \AA, 1.764 \AA, 1.841 \AA, 2.017 \AA, 2.065 \AA$, and $2.494 \AA$ for $\operatorname{Mo}(3)$. As a result, the mean distance to the four nearest oxygen atoms in $\gamma-\mathrm{CuMoO}_{4}$ is equal to $\sim 1.82 \AA$ with a standard 
deviation of $0.11 \AA$, which is twice as large as that in $\alpha-\mathrm{CuMoO}_{4}$. Thus, the local environment of molybdenum atoms is more distorted in the $\gamma$-phase.

Our results in Figure 4 suggest that the mean Mo-O distance estimated from the position of the RDF maximum for the group of four shortest Mo-O bonds is equal to $1.76 \pm 0.02 \AA$ and remains constant at all temperatures in both phases. However, the amplitude and width of the RDF peak vary following the $\gamma$-to- $\alpha$ phase transition. Note that the RDF determined by EXAFS includes both thermal disorder and static distortions, and EXAFS is also more sensitive to shorter distances. Therefore, some small differences exist in the mean Mo-O distances for the group of four nearest oxygen atoms observed by EXAFS in the present work and by diffraction in Ref. ${ }^{1}$.

A parametrization of the obtained RDFs (Figure 4) using a proper analytical model can be performed to quantitatively estimate the variation in the local environment upon the phase transition. When the Gaussian model is applied, the amplitude of the RDF peak is given by $N / \sqrt{2 \pi \sigma^{2}}$, where $N$ is the coordination number, and $\sigma^{2}$ is the mean-square relative displacement (MSRD) factor describing both static and thermal disorder. In our case, the coordination number is known $(N=4)$; therefore, the temperature-dependence of the MSRD factor $\sigma^{2}$ for the group of four nearest oxygen atoms can be calculated and is shown in Figure 5. Such procedure allowed us to reduce the influence of the RDFs shape (including peak asymmetry and spurious peaks) on the MSRD values.

One can expect that the MSRD factor $\sigma^{2}$ will increase upon heating due to the larger amplitude of thermal vibrations. However, the variation of the MSRD in the $\gamma$-phase below $225 \mathrm{~K}$ is negligible being within the error bars due to the dominant role of static distortions.

The thermal disorder contribution to the MSRD of Mo-O bonds was estimated by performing lattice dynamics calculations using the GULP $\operatorname{code}^{25}$ for scheelite structure type calcium molybdate $\left(\mathrm{CaMoO}_{4}\right)$, in which molybdenum atoms have tetrahedral coordination by oxygen atoms with the bond length $R(\mathrm{Mo}-\mathrm{O})=1.775 \AA,{ }^{26}$ close to that in $\alpha-\mathrm{CuMoO}_{4}$. The MSRD $\sigma^{2}$ for the Mo-O atom pair was evaluated in the harmonic approximation from the eigenfrequencies and eigenvectors of the dynamical matrix through numerical integration over all normal modes. ${ }^{27,28}$ We used a force-field model similar to that successfully validated by us previously for several tungstates with the scheelite structure ${ }^{29}$ but re-optimized to reproduce the crystal lattice parameters and elastic constants of $\mathrm{CaMoO}_{4} \cdot{ }^{26} \mathrm{The}$ 
MSRD values $\sigma^{2}(\mathrm{Mo}-\mathrm{O})=0.00042 \AA^{2}$ and $0.00128 \AA^{2}$ were obtained at $10 \mathrm{~K}$ and $300 \mathrm{~K}$, respectively. Comparison of these small MSRD values with those reported in Figure 5 for short Mo-O bonds supports our conclusion on the dominant role of the static disorder in both $\mathrm{CuMoO}_{4}$ phases.

The significant decrease of the MSRD factor from $\sim 0.010 \AA^{2}$ to $\sim 0.005 \AA^{2}$ above $225 \mathrm{~K}$ (Figure 5) indicates the occurrence of a phase transition and a transformation of the local environment of molybdenum atoms from strongly distorted octahedral in $\gamma-\mathrm{CuMoO}_{4}$ to less distorted tetrahedral in $\alpha-\mathrm{CuMoO}_{4}$. This gradual transition occurs within a temperature range of 225-275 K. Similar results were found in previous studies using differential scanning calorimetry, magnetic susceptibility, optical absorption and reflectivity measurements. ${ }^{4,30,31}$

\section{Conclusion}

The thermochromic $\gamma$-to- $\alpha$ phase transition in $\mathrm{CuMoO}_{4}$ was studied by Mo K-edge extended X-ray absorption fine structure spectroscopy in the temperature range of 10 to $300 \mathrm{~K}$. The radial distribution functions within the first coordination shell of molybdenum atoms were reconstructed by the regularization-like method. ${ }^{13}$ Such a model independent approach allowed us to smoothly follow the change of the molybdenum coordination from octahedral to tetrahedral upon temperature increase.

The influence of thermal effects on the local environment around molybdenum atoms in both the $\gamma$ and $\alpha$ phases is rather weak, and the disorder is dominated by static distortions. Upon the $\gamma$-to$\alpha$ phase transition, the molybdenum coordination by oxygen atoms changes from strongly distorted octahedral to less distorted tetrahedral. The temperature dependence of the radial distribution function suggests that the transition occurs gradually within the two phase coexistence range of 225-275 K, in agreement with the results of previous studies. ${ }^{4,30,31}$

Acknowledgements The work was supported by philanthropist MikroTik and administrated by the University of Latvia Foundation. The experiment at HASYLAB/DESY was performed within the project I-20160149 EC.

Conflict of Interest The authors declare no conflict of interest. 
[1] M. Wiesmann, H. Ehrenberg, G. Miehe, T. Peun, H. Weitzel, H. Fuess, J. Solid State Chem. 1997, 132,88 .

[2] F. Rodríguez, D. Hernández, J. Garcia-Jaca, H. Ehrenberg, H. Weitzel, Phys. Rev. B 2000, 61, 16497.

[3] G. Steiner, R. Salzer, W. Reichelt, Fresenius J. Anal. Chem. 2001, 370, 731.

[4] M. Gaudon, C. Carbonera, A. E. Thiry, A. Demourgues, P. Deniard, C. Payen, J. F. Létard, S. Jobic, Inorg. Chem. 2007, 46, 10200.

[5] L. Robertson, M. Gaudon, S. Jobic, P. Deniard, A. Demourgues, Inorg. Chem. 2011, 50, 2878.

[6] L. Robertson, N. Penin, V. Blanco-Gutierrez, D. Sheptyakov, A. Demourgues, M. Gaudon, J. Mater. Chem. C 2015, 3, 2918.

[7] V. Blanco-Gutierrez, L. Cornu, A. Demourgues, M. Gaudon, ACS Appl. Mater. Inter. 2015, 7, 7112.

[8] H. Ehrenberg, H. Weitzel, H. Paulus, M. Wiesmann, G. Wltschek, M. Geselle, H. Fuess, J. Phys. Chem. Solids 1997, 58, 153.

[9] T. Ito, H. Takagi, T. Asano, Chem. Mater. 2009, 21, 3376.

[10] S. C. Abrahams, J. L. Bernstein, P. B. Jamieson, J. Chem. Phys. 1968, 48, 2619.

[11] D. Hernández, F. Rodrıguez, J. Garcia-Jaca, H. Ehrenberg, H. Weitzel, Physica B 1999, $265,181$.

[12] I. Jonane, A. Cintins, A. Kalinko, R. Chernikov, A. Kuzmin, Fiz. Nizk. Temp. 2018, 44, 568.

[13] A. Kuzmin, J. Purans, J. Phys.: Condens. Matter 2000, 12, 1959.

[14] A. Kuzmin, J. Chaboy, IUCrJ 2014, 1, 571.

[15] B. Ravel, M. Newville, J. Synchrotron Rad. 2005, 12, 537.

[16] J. Timoshenko, A. Kuzmin, J. Purans, J. Phys.: Condens. Matter 2014, 26, 055401.

[17] I. Jonane, K. Lazdins, J. Timoshenko, A. Kuzmin, J. Purans, P. Vladimirov, T. Gräning, J. Hoffmann, J. Synchrotron Rad. 2016, 23, 510.

[18] A. Anspoks, A. Kalinko, J. Timoshenko, A. Kuzmin, Solid State Commun. 2014, 183, 22. 
[19] A. Kuzmin, A. Anspoks, A. Kalinko, J. Timoshenko, R. Kalendarev, Sol. Energy Mater. Sol. Cells 2015, 143, 627.

[20] P. A. Lee, P. H. Citrin, P. Eisenberger, B. M. Kincaid, Rev. Mod. Phys. 1981, 53, 769.

[21] A. L. Ankudinov, B. Ravel, J. J. Rehr, S. D. Conradson, Phys. Rev. B 1998, 58, 7565.

[22] J. J. Rehr, R. C. Albers, Rev. Mod. Phys. 2000, 72, 621.

[23] L. Hedin, B. I. Lundqvist, J. Phys. C: Solid State Phys. 1971, 4, 2064.

[24] A. Kuzmin, R. Grisenti, Phil. Mag. B 1994, 70, 1161.

[25] J. D. Gale, A. L. Rohl, Mol. Simul. 2003, 29, 291.

[26] A. Senyshyn, H. Kraus, V. B. Mikhailik, L. Vasylechko, M. Knapp, Phys. Rev. B 2006, 73, 014104.

[27] J. S. Chung, M. F. Thorpe, Phys. Rev. B 1997, 55, 1545.

[28] E. R. Cope, M. T. Dove, J. Appl. Crystallogr. 2007, 40, 589.

[29] A. Kalinko, M. Bauer, J. Timoshenko, A. Kuzmin, Phys. Scr. 2016, 91, 114001.

[30] M. Gaudon, P. Deniard, A. Demourgues, A. E. Thiry, C. Carbonera, A. Le Nestour, A. Largeteau, J. F. Létard, S. Jobic, Adv. Mater. 2007, 19, 3517.

[31] A.-E. Thiry, M. Gaudon, C. Payen, N. Daro, J.-F. Létard, S. Gorsse, P. Deniard, X. Rocquefelte, A. Demourgues, M.-H. Whangbo, S. Jobic, Chem. Mater. 2008, 20, 2075. 


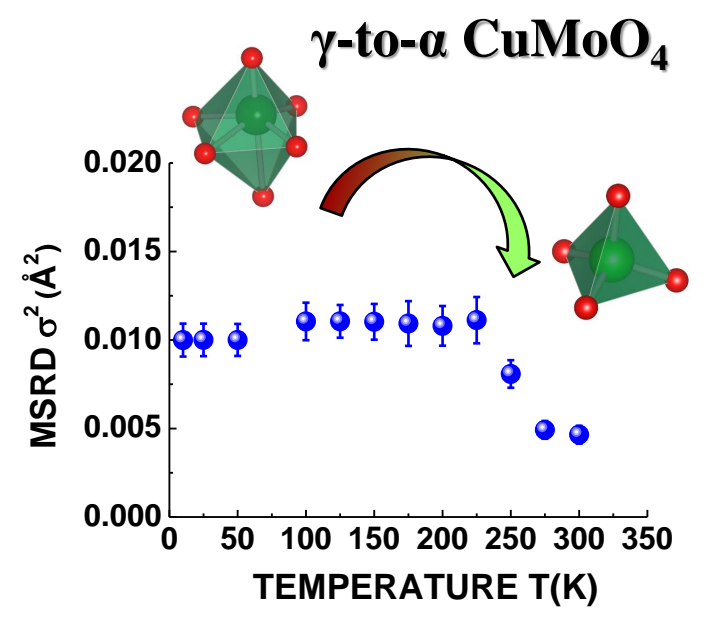

The thermochromic $\gamma$-to- $\alpha$ phase transition in $\mathrm{CuMoO}_{4}$ was studied by Mo K-edge extended X-ray absorption fine structure spectroscopy in the temperature range of 10 to $300 \mathrm{~K}$. The local atomic structure around molybdenum atoms changes upon the transition during heating from strongly distorted octahedral to less distorted tetrahedral coordination. The temperature dependence of the radial distribution function suggests that the transition occurs gradually within the two-phase coexistence range of $225-275 \mathrm{~K}$.

\section{Graphical Table of Contents}

